acta medica

\title{
Liposomal Amphotericin B Induced Acute Reactions
}

Melda Bahap', [MSc]
ORCID: 0000-0003-1392-1135

Pinar Bakir Ekinci', [Pharm]

ORCID: 0000-0003-0694-6078

Sehnaz Alp ${ }^{2}$, [MD]

ORCID: 0000-0001-9424-8346

Serife Gul Oz $z^{3}$, [MD]

ORCID: 0000-0002-5197-445X

Kutay Demirkan', [MD]

ORCID: 0000-0002-6427-5826

This study has not been presented in any congress or symposium previously.

'Hacettepe University, Faculty of Pharmacy, Department of Clinical Pharmacy, Ankara, Turkey.

${ }^{2}$ Hacettepe University, Faculty of Medicine, Department of Infectious Diseases and Clinical Microbiology, Ankara, Turkey.

${ }^{3}$ Hacettepe University, Faculty of Medicine, Department of Internal Medicine, Ankara, Turkey.

Corresponding Author: Melda Bahap

Hacettepe University Faculty of Pharmacy, Department of Clinical Pharmacy, Sıhhıye/ Ankara, Turkey.

E-mail: melda_610@hotmail.com

https://doi.org/10.32552/2021.ActaMedica.566

\section{sel ABSTRACT Cen}

Three formulations of amphotericin B are available: liposomal, lipid complex and conventional. The liposomal amphotericin B is more preferred agent than other formulations because of its tolerability, safety and potent antifungal activity. However, the liposomal amphotericin B can cause infusion-related reactions. In this case report, we aimed to report a patient who developed infusion-related reactions during the treatment with the liposomal amphotericin B but eventually tolerated the prolonged infusion. In this case report, we present a patient who developed an infusion-related reaction during The liposomal amphotericin B treatment. A 26-year-old male patient with acute promyelocytic leukemia was hospitalized for the third course of chemotherapy. Due to the invasive fungal infection history in previous hospitalizations, the liposomal amphotericin B $400 \mathrm{mg}$ (IV, $5 \mathrm{mg} / \mathrm{kg}$ ) once daily was initiated as secondary antifungal prophylaxis. Swelling in infusion site and chest pain were reported within 10 minutes of the liposomal amphotericin $B$ administration, and the infusion rate was slowed down to $400 \mathrm{mg} / 6$ hours from $400 \mathrm{mg} / 2$ hours. All these reactions disappeared with prolonged infusion time. The patient received a total of 7 liposomal amphotericin B doses subsequently without any reaction during the chemotherapy cycle. In our experience, the liposomal amphotericin B-induced infusion-related reactions can be resolved by prolonging the infusion time.

Keywords: Liposomal amphotericin B, infusion-related reactions, amphotericin $B$ deoxycholate, infusion rate.

Received: 15 February 2021, Accepted: 27 February 2021,

Published online: 20 November 2021 


\section{INTRODUCTION}

Amphotericin B is available in formulations of liposomal, lipid complex and conventional. The first three formulations are lipid derivatives more widely used than amphotericin B deoxycholate because of their better tolerability and safety [1]. Liposomal amphotericin B (LAB) is a broadspectrum antifungal agent that consists of $a$ single bilayer liposomal drug delivery system [1, 2]. Reactions to amphotericin $B$ infusion include nausea, vomiting, fever, chills, and cardiopulmonary events such as chest pain, dyspnea, flushing and hypotension. These reactions usually occur at the onset of infusion and dependent on the rate, but not related to the dose [3]. In a study by Walsh et al., it was found that infusion-related reactions caused by $L A B$ were significantly lower than amphotericin B deoxycholate [4]. Collazos et al. argued that the incidence of amphotericin $B$ deoxycholate-associated pulmonary reactions was higher than amphotericin B lipid complex because of faster infusion rate ( 2 hours versus 4-6 hours) [1]. In this case report, it was reported a patient who developed infusion-related reactions with $L A B$ but eventually tolerated the prolonged infusion.

\section{CASE PRESENTATION}

A 26-year-old, $80 \mathrm{~kg}$, male patient was admitted to the hospital due to the complaints of swelling, increase in fever and pain in his right lower leg and was diagnosed with deep vein thrombosis (DVT). The patient had no comorbidities and allergy history at the time of admission. Enoxaparin was started for DVT treatment. Upon detection of pancytopenia during hospitalization, the patient was consulted with hematologists and diagnosed with acute promyelocytic leukemia. A combination treatment with idarubicin and all-trans retinoic acid (ATRA) were initiated.

Prophylactic antimicrobial regimen with posaconazole (oral, $300 \mathrm{mg}$ daily after initial 300 $\mathrm{mg}$ twice a day loading dose on the first day), levofloxacin (oral, $750 \mathrm{mg}$ daily) and valaciclovir (oral, $500 \mathrm{mg}$ twice daily) were also started. On his follow-up, neutropenic fever was developed in patient. Meropenem (intravenous [IV], $1000 \mathrm{mg}$ three times daily) and teicoplanin (IV, a loading dose of $12 \mathrm{mg} / \mathrm{kg}$ administered 3 times at $12 \mathrm{~h}$ intervals, followed by a maintenance dose of $12 \mathrm{mg} / \mathrm{kg}$ daily) were added empirically and for severe mucositis, respectively. On the fifth day of antimicrobial treatment, serum galactomannan index was negative and no growth in fungal cultures, but he was still febrile and computed tomography (CT) of the chest revealed signs of fungal infection. Posaconazole was discontinued and LAB $400 \mathrm{mg}$ (IV, $5 \mathrm{mg} / \mathrm{kg}$ ) once daily was started for the treatment of invasive pulmonary fungal infection. His status improved, and he recovered from neutropenia. On the fourth week of $L A B$ treatment, repeated chest $C T$ indicated resolution of fungal infection. $L A B$ was stopped and the patient was discharged.

One month later, he was readmitted to the hospital to receive the next chemotherapy course. Due to the prior invasive fungal infection history, $L A B$ $400 \mathrm{mg}$ (IV, $5 \mathrm{mg} / \mathrm{kg}$ ) once daily was initiated as secondary antifungal prophylaxis. However, chest pain and swelling in infusion site were reported within 10 minutes of $L A B$ administration on the 2 nd day of treatment. $L A B$ infusion was stopped because infusion-related reaction was suspected. No therapy was given to the patient to relieve infusion-related reactions. After the improvement of patient's symptoms, LAB treatment was restarted by slowing the infusion rate from $400 \mathrm{mg} / 2$ hours to $400 \mathrm{mg} / 6$ hours. All these reactions disappeared with prolonged infusion. The patient received a total of 7 LAB doses subsequently without any reaction during the chemotherapy cycle. Thereafter, $L A B$ administration with prolonged infusion during his next chemotherapy course was also completed without any reactions.

\section{DISCUSSION}

In this case report, we present a patient who received 2 hours infusion of $400 \mathrm{mg}$ (IV, 5mg/ $\mathrm{kg}$ ) $L A B$ and developed infusion-related adverse reactions including swelling in the infusion site and chest pain. These reactions disappeared when the infusion rate was prolonged to 6 hours.

The use of conventional amphotericin B is restricted by the high incidence of infusion-related adverse events and nephrotoxicity. Alternative formulations have been developed to minimize 
the toxicity and adverse effects and to increase the therapeutic index of the drug [5]. Published safety data provided by the manufacturer and data from a multicenter phase II/III study evaluating over 100 treatment episodes with LAB showed increased tolerability and significantly reduced toxicity compared with conventional amphotericin $B$ [6]. However, in the literature, infusion-related adverse reactions with $L A B$ have been rarely reported [7]. A possible relationship between the infusion rate of $L A B$ and adverse effects has not been studied sufficiently. Animal studies have shown that amphotericin B directly damages endothelial cells in a dose and time-dependent manner, irrespective of the vehicle (deoxycholate) [8]. It also alters neutrophil functions [9], induces neutrophil-independent lung damage associated with oxidative stress and eicosanoid production [10], causes lung dysfunction through the release of cyclooxygenase products of arachidonic acid metabolism [11, 12].

In a case series, LAB was administered at a dose of $3 \mathrm{mg} / \mathrm{kg} /$ day over 1 -hour infusion in 3 patients. The first patient developed chest tightness and difficulty of breathing, the second patient developed dyspnea and acute hypoxia 10 minutes after the onset of the infusion. The third patient developed chest pain at the fifth minute of the infusion. Two of these patients tolerated $L A B$ when infusion rate was extended to 2 hours and with administration of meperidine. To avoid adverse reactions, at least two hours of $L A B$ infusion was recommended [11]. Package insert of $L A B$ approved by the Food and Drug Administration (FDA) had a few reports of chest pain, flushing, back pain with or without chest tightness after LAB administration. However, infusion-related reactions were reported to be very rare, mostly developed shortly after the onset of infusion and disappeared with slower infusion rates [13].

It is recommended that the first infusion of $L A B$ should be administered slowly under observation, and the patient should be specifically asked about the presence of even mild symptoms on the first days of treatment. If acute respiratory symptoms develop during administration of $L A B$, the infusion should be stopped immediately to prevent further clinical deterioration. If treatment with $\angle A B$ is to be continued, the patient should be closely monitored for re-exposure, the infusion rate should be slowed down and symptomatic treatment should be considered if necessary [1]. However, the exact duration of the slow infusion rate is not clear. The product information reported by FDA suggested that 60 minutes infusion rate could be initiated for patients who could tolerate treatment with 120 minutes [13]. In a study by Schöffski et al., any difference was not observed between 1 and 4 hours of infusion time in terms of pulmonary toxicity [14]. Collazos et al. reported that no further symptoms were developed when the infusion time was increased to 6 hours in a patient who had symptoms while receiving amphotericin $B$ lipid complex ( $3 \mathrm{mg} / \mathrm{kg} /$ day) over for 2 hours [1].

In our patient, infusion-related reactions developed shortly after the onset of LAB infusion. Based on studies by Schöffski et al. [14], and Collazos et al. [1], we extended the infusion time to 6 hours instead of 2 hours. The symptoms disappeared when the infusion rate was slowed down.

In summary, infusion-related reactions with $L A B$ appear to occur rarely. However, these symptoms can sometimes be life-threatening. Even though some reports indicate that the infusion rate does not provide relief from symptoms whether the drug was given in 1 hour or 4 hours, we recommend based on our case, infusion of LAB be over 6 hours and careful monitoring was provided.

\section{Key Points}

Even though infusion-related reactions with liposomal amphotericin B appear to occur rarely, these symptoms can sometimes be life-threatening.

Some reports indicate that the infusion rate does not provide relief from symptoms whether the drug was given in 1 hour or 4 hours.

In this study, the infusion of LAB over the course of 6 hours was recommended to remain on the safe side with careful monitoring.

\section{CONFLICT Of INTEREST STATEMENT}

No, there is no conflict of interest that I should disclose, having read the above statement. 
[1] Collazos J, Martinez E, Mayo J, et al. Pulmonary reactions during treatment with amphotericin $\mathrm{B}$ : review of published cases and guidelines for management. Clin Infect Dis 2001; 33(7): E75-82.

[2] Hagihara M, Yamagishi $Y$, Hirai J, et al. Drug-induced hypersensitivity syndrome by liposomal amphotericin-B: a case report. BMC Res Notes 2015; 8: 510.

[3] Goodwin SD, Cleary JD, Walawander CA, et al. Pretreatment regimens for adverse events related to infusion of amphotericin B. Clin Infect Dis 1995; 20(4): 755-61.

[4] Walsh TJ, Finberg RW, Arndt C, et al. Liposomal amphotericin B for empirical therapy in patients with persistent fever and neutropenia. National Institute of Allergy and Infectious Diseases Mycoses Study Group. N Engl J Med 1999; 340(10): 764-71.

[5] Tollemar J, Ringden O. Lipid formulations of amphotericin B. Less toxicity but at what economic cost? Drug Saf 1995; 13(4): 207-18.

[6] Meunier F, Prentice HG, Ringden O. Liposomal amphotericin B (AmBisome): safety data from a phase II/ III clinical trial. J Antimicrob Chemother 1991; 28 Suppl B: 83-91.

[7] Arning $M$, Heer-Sonderhoff $A H$, Wehmeier $A$, et al. Pulmonary toxicity during infusion of liposomal amphotericin B in two patients with acute leukemia. Eur J Clin Microbiol Infect Dis 1995; 14(1): 41-3.
[8] Cutaia M, Bullard SR, Rudio $\mathrm{K}$ et al. Characteristics of amphotericin B-induced endothelial cell injury. J Lab Clin Med 1993; 121(2): 244-56.

[9] Sullivan GW, Carper HT, Mandell GL. Pentoxifylline modulates activation of human neutrophils by amphotericin B in vitro. Antimicrob Agents Chemother 1992; 36(2): 408-16.

[10] McDonnell TJ, Chang SW, Westcott JY, et al. Role of oxidants, eicosanoids, and neutrophils in amphotericin B lung injury in rats. J Appl Physiol 1988; 65(5): 2195-206.

[11] Johnson MD, Drew RH, Perfect JR. Chest discomfort associated with liposomal amphotericin B: report of three cases and review of the literature. Pharmacotherapy 1998; 18(5): 1053-61.

[12] Gigliotti F, Shenep JL, Lott L, et al. Induction of prostaglandin synthesis as the mechanism responsible for the chills and fever produced by infusing amphotericin B. J Infect Dis 1987; 156(5): 784-9.

[13] Food and Drug Administration. AmBisome ${ }^{\oplus}$ (amphotericin B) liposome for injection. https://www.accessdata.fda. gov/drugsatfda_docs/label/2008/050740s016lbl.pdf (accessed June 2019).

[14] Schoffski P, Freund M, Wunder R, et al. Safety and toxicity of amphotericin B in glucose $5 \%$ or intralipid $20 \%$ in neutropenic patients with pneumonia or fever of unknown origin: randomised study. BMJ 1998; 317(7155): 379-84. 